\title{
A Multiscale Analysis Model Applied to Natural Surfaces
}

\author{
Frédéric FALZON Gérard GIRAUDON Marc BERTHOD
}

INRIA, BP.93, F-06902 SOPHIA ANTIPOLIS Cedex

\begin{abstract}
Smoothing (regularization), interpolation and surface reconstruction are well known subjects in computer vision. The major difficulty is to choose a model well suited for one of these goals and driven by a minimum number of parameters. Another problem also arises when we want to do one of these operations adaptatively, i.e. local features are processed in keeping with the application domain (e.g. cartography). Our goal is to present a discrete operator driven by only one parameter, allowing both global and local processing of a surface and, well suited to smoothing, interpolation and surface reconstruction.
\end{abstract}

\section{Introduction}

In this paper we present a discrete adaptative surface model well suited to the problem of surface regularization and reconstruction. This model is based on a well known analog network analogy and, we show that, adaptativity is given by its ability to change its behaviour without modifying its intrinsic structure. A more complete study of this model has been previously given in [4] and [5] but here we focus more on applications. Until now, a lot of works have been published in reconstruction and interpolation of surfaces $[6,2,1,14,15,13,12]$. Our contribution is focussed on the framework of DEM (Digital Elevation Model) processing and based on the description of a model and a discrete operator driven by a minimum number of parameters (only one).

\section{A discrete scaled operator}

Models based on analog networks have been already used in various applications $[11,7,8,9]$. Our goal is to show that, from this kind of model, we can derive an operator to solve both smoothing from sparse data and surface reconstruction from relevant 2D structures. In the following we give the expression of this operator, then we give two examples. (The complete description of the model from which we obtain our operator is given in $[4,5])$.

Let $\Omega$ be a set of nodes $p_{i}$ distributed on a line (1D case), we note

$\mathcal{V}\left(p_{i}\right)=\left\{p_{k} \in \Omega,|i-k|=1\right\}$, the neighbourhood of the node $p_{i}$;

int $\Omega=\left\{p_{i} \in \Omega, \mathcal{V}\left(p_{i}\right) \subset \Omega\right\}$, the interior of the set $\Omega$;

$\partial \Omega=\Omega-$ int $\Omega$, the boundary of $\Omega$;

$1_{\Omega}$, the indicator function of the set $\Omega$;

Let $\alpha_{i}^{j} \in \mathbb{R}^{+}$the coefficient which connect two points $i$ and $j$ and $\Gamma_{a} \in \mathbb{R}^{N \times N}$ the finite matrix given by

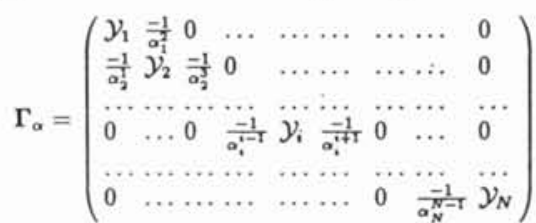

$$
\text { with } \mathcal{Y}_{i}=1+\sum_{k \in \mathcal{V}\left(p_{i}\right)} \frac{1}{\alpha_{i}^{k}} \text {. }
$$

This operator will appear in a linear system $V=\Gamma_{\alpha}^{-1} \hat{V}$, where $\tilde{V}$ is a given vector. In the $2 \mathrm{D}$ case $\hat{V}$ is an image stored in a vector form and $V$ is the resulting transformed image.

\subsection{First case - Smoothing operator}

We consider an isotropic distribution of $\alpha$, i.e. we make the assumption : $\alpha_{i}^{k}=\alpha_{k}^{i}=\alpha=$ Const $\forall i, k \in$ int $(\Omega)$. In this case the discrete operator $\Gamma_{\alpha}^{-1}$ is contractive i.e. it decreases the norm of all vector it is applied to (see [5]). In a geometric point of view, this operator reduce the curvature of all discrete function (vector). Then we can solve the problem of smoothing from sparse data. We replace the previous 1D operator by its 2D analogous, and we consider 4-connectivity. Let $\vec{V}$ be a $2 \mathrm{D}$ discrete function. The value of the grey level of the resulting image, called $V$ is given by

$$
\begin{aligned}
V_{(i, j)}= & \tilde{V}_{(i, j)}-\sum_{k=j-1, k \neq j}^{j+1} \frac{1}{\alpha}\left(V_{(i, j)}-V_{(i, k)}\right) \\
& -\sum_{k=i=1, k \neq i}^{i+1} \frac{1}{\alpha}\left(V_{(i, j)}-V_{(k, j)}\right)
\end{aligned}
$$

An example of smoothing is given in fig( 3 ). The data test given in fig $(3)(a)$ are voluntary the same as [13] to compare the results. The final solution in fig $(3)(\mathrm{g})$ is very closed to the solution obtain by [13] with thin plate interpolant.

\subsection{Second case : Reconstruction operator}

We consider an anisotropic distribution of $\alpha$, i.e. $\alpha_{i}^{k} \neq \alpha_{k}^{i} \quad \forall i \neq$ $k$. In this case, it is possible to put asymmetrical weights between two points and therefore, the evolution law of the points are not identical. Consider the following scheme for natural surface reconstruction

[1] Take $\mathcal{D}$ the set of all points that belong to ridge lines or stream networks (assumed to be given by an external process);

[2] Two points $i$ and $j$ are connected by $\alpha_{j}^{i}$ in one direction and by $\alpha_{i}^{i}$ in the reverse direction. if we put $\alpha_{j}^{i} \neq \alpha_{i}^{i}$ one can influence a point more or less, according to the value affected to the corresponding parameter. If we want a point $i$ not to be influenced by others, but it should itself influence the others, we take $\alpha_{j}^{i}=\infty$ and $\alpha_{i}^{j}=\alpha$ : then the point $i$ remains the same for all values of $\alpha$, i.e. the location and altitude are preserved across the reconstruction.

[3] When all parameters are initialized, we solve the linear system $\Gamma_{\alpha} V=\tilde{V}$ for increasing values of $\alpha$.

We choose to fix only ridge lines, stream networks and boundary points, all the others are left to 0 (no information). Figs $(4)(a, b)$ show the 3D representation of the original DEM, and the points that have to be fixed to make the reconstruction. 
These points are obtained with the algorithm described in [3]. Figs (4) $(c, d, e, f)$ show the reconstruction obtained for different numbers of iterations (we solve $\Gamma_{\alpha} V^{n+1}=V^{n}$ with $V^{0}=\tilde{V}$ ). In figs $(4)(\mathrm{g}, \mathrm{h})$ we give the contour lines of the original and reconstructed DEM. These results are obtained by considering all surface points for the initialization of the algorithm. Some points are fixed (as in the first case) and the others are initialized with the original data. This leads to adopt a less brutal approach than in the first case where we have considered that no information is equivalent to 0 . We can see that valleys are narrowed and the relief is more accentuated, but spatial morphology is relatively well conserved despite the smoothing.

\subsection{A remark about fixed point across scales}

In [4] we show that if $\alpha$ is the same between all points $\Gamma_{\alpha}$ is the discretization of the heat equation with certain boundary conditions (the 1D beat equation is $f_{t}=\zeta f_{x x}$ ). Suppose we are in the ID case, then fixing a point with $\alpha_{j}^{i}=\infty$ and $\alpha_{i}^{j}=\alpha$ comes to put a 1 on the diagonal element of the line $i$ of $\Gamma_{a}$ and 0 elsewhere on this line, that is

$$
\mathbf{\Gamma}_{\alpha}=\left(\begin{array}{cccccccccc}
\ldots & \ldots & \ldots & \ldots & \ldots & \ldots & \ldots & \ldots & \ldots & \ldots \\
0 & \ldots & \frac{-1}{a} & \ddot{y}_{i-1} & \frac{-1}{a} & \ldots & \ldots & \ldots & \ldots & 0 \\
0 & \ldots & \ldots & 0 & 1 & 0 & \ldots & \ldots & \ldots & 0 \\
0 & \ldots & \ldots & \ldots & \frac{-1}{a} & y_{i+1} & \frac{-i}{a} & 0 & \ldots & 0 \\
\ldots & \ldots & \ldots & \ldots & \ldots & \ldots & \ldots & \ldots & \ldots & \ldots
\end{array}\right)
$$

This leads to an uncoupled system and this problem can be solved piecewise. However there may exist discontinuities of the first derivative on frontier points of each subdomain because the stationnary solution tends to be linear between two boundary points (see fig (1)). Suppose that $\mathcal{D}$ is the domain that includes each solution (noted $f^{\mathcal{D}}$ ) computed in each separate subdomain $\mathcal{D}_{i}$ of $\mathcal{D}$. Each of these $f^{\mathcal{D}}$ is obtained by solving the problem on each subdomain delimited by fixed points (without considering the natural boundary points $\partial \Omega$ ). Then $\mathcal{D} \in$ int $\Omega, \mathcal{D}=\bigcup \mathcal{D}_{i}$ is a set of contiguous domains and we can write the global solution as $f^{D}=\sum_{k=1}^{C a r d \mathcal{D}} f^{\mathcal{D}_{*}} \mathbf{1}_{\mathcal{D}_{k}}$, where each $f^{\mathcal{D}_{k}}$ is solution of the following problem : assuming that the space variable $x$ belongs to $[0,1]$ in each subdomain, $\zeta$ the diffusion coefficient and $\tilde{f}$ the initial condition, we have to solve $f_{t}^{D_{k}}=\zeta f_{x_{*}}^{D_{k}}$ with conditions $f^{D_{*}}(0, t)=f^{D_{*}}(0)$ on $\partial_{0}^{D_{\star}}$ and $f^{\mathcal{D}_{*}}(1, t)=f^{\mathcal{D}_{\star}}(1)$ on $\partial_{1}^{\mathcal{D}_{\star}}$.

Now if we want that on frontier points the solution belongs to $C^{1}$ we have to introduce new conditions at these points. Suppose we want a null first derivative on frontier points. Then the problem has to be solved with four boundary conditions and this is impossible because the degree of the partial differential equation is two. Then we consider the following problem where we act on the curvature of the initial condition instead of the initial condition itself

$$
f_{t}^{\mathcal{D}_{*}}=-\zeta f_{\text {xxx }}^{\mathcal{D}_{k}}
$$

with conditions $f^{\mathcal{D}_{\star}}(0, t)=\tilde{f}^{\mathcal{D}_{k}}(0)$ on $\partial_{0}^{\mathcal{D}_{\star}}, f^{\mathcal{D}_{\star}}(1, t)=\tilde{f}^{\mathcal{D}_{\star}}(1)$ on $\partial_{1}^{D_{*}}, \frac{\partial f^{D_{*}}(x, t)}{\partial_{x}}=0$ on $\partial_{0}^{D_{*}}$ and $\partial_{1}^{\mathcal{D}_{*}}$. Here $\partial_{0}^{\mathcal{D}_{*}}$ and $\partial_{1}^{D_{*}}$ denotes the left and right boundary. This last equation has been evoked in [10](pp.11). Numerically, the connection between the scale parameter of this model and the first one (analog network) is straightforward by putting $\alpha=\zeta^{-1} \frac{\Delta t}{\Delta}$. The boundary conditions are inserted in the new matrix $\vec{\Gamma}_{\alpha}^{\prime}$ by putting Neumann conditions (null derivative) in the first and last line, and Dirichlet conditions (fixed points) in the second and last but one line. In the initial case the numerical scheme was given by $\Gamma_{\alpha}=I-\alpha^{-1} \Delta$ and here $\Gamma_{\alpha}=I+\alpha^{-1} \Delta^{2}$ (here $\Delta$ is the laplacian). One can see here the utility to consider the problem on a finite domain. In dimension two this problem is different because the constraints are 2D primitives (e.g. ridge lines), not inevitably closed and, in this case, it is not always possible to make piecewise computations.

\section{Conclusions}

We have presented a discrete operator which results from an electrical analogy (see $[4,5])$. By making two different assumptions on the neighbourhood relations, we proposed two applications based on the use of the same operator. The first concerns smoothing from sparse data and the second concerns natural surface reconstruction. The results are encouraging and show the ability of this operator to take into account different neighbourhood relations. Moreover parallel implementation can be envisaged, due to the discrete nature and the structure of this operator.

\section{Acknowledgments}

We would like to thanks N.Maman, P.Leymarie, S.Mathieu J.M.Malé and B.Vasselle for their help in some parts of this study.

\section{References}

[1] A. Blake et A. Zisserman Visual Reconstruction, MIT Press Cambridge MA 1987.

[2] T.E. Boult, J.R. Kender Visual surface reconstruction using sparse depth data, in Proc CVPR pp 68-76, 1986.

[3] J. Fairfield, P. Leymarie Drainage Networks From Grid Digital Elevation Models Water Resources Research, Vol.27, No.5 Pages 709-717, May 91.

[4] F. Falzon, G. Giraudon, M. Berthod Vers un Modèle pour l'Analyse Multiéchelle de Surface SD INRIA Report No 1639, 1992.

[5] F. Falzon, G. Giraudon, M. Berthod A Multiscale Analysis Model Applied to Natural Surfaces IEEE Workshop on Applications of Computer Vision. Palm Springs, 1992.

[6] W.E.L. Grimson A Computational Study of the Human Early Visual System, MIT Press 1981.

[7] B.K.P. Horn Robot Vision The MIT Press McGraw-Hill Book company - 1986.

[8] A. Lumsdaine, J.L. Wyatt, L.M.Elfadel Nonlinear Analog Networks for Image Smoothing and Segmentation MIT Artificial Intelligence Laboratory. A.I. Memo No. 1280 - Jan 91.

[9] M. Mahowald, C. Mead Une rétine en silicium Pour la Science - No 165 - Jul 91.

[10] T.Poggio, V.Torre, C.Koch An Analog Model of Computation For the IIl-Posed Problems of Early Vision MIT - AI Laboratory, A.I.Memo 783, C.B.I.P Paper 002, May 84

[11] T.Poggio, V.Torre, C.Koch Computational Vision and Regularization Theory Nature Vol.317. 26 Sept 1985

[12] S.S. Sinha, B.G. Schunck Surface Approximation Using Weighted Splines, in Proc CVPR Hawai Jun 91.

[13] R. Szeliski Fast surface interpolation using hierarchical basis function, IEEE PAMI, Vol 12(6), pp 513-528, Jun 90.

[14] D. Terzopoulos The Computation of visible-surface representations IEEE Trans on PAMI Vol 10(4) pp 417-438, 1988.

[15] D. Terzopoulos, M.Vasilescu Sampling and Reconstruction with Adaptative Meshes, in Proc CVPR Hawai 91. 


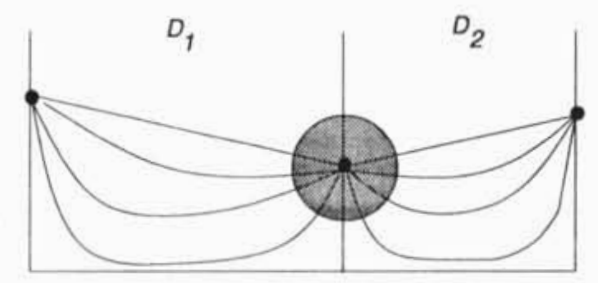

Figure 1: Two connected subdomains. At the connection point (in the circle) the solution is not $C^{1}$

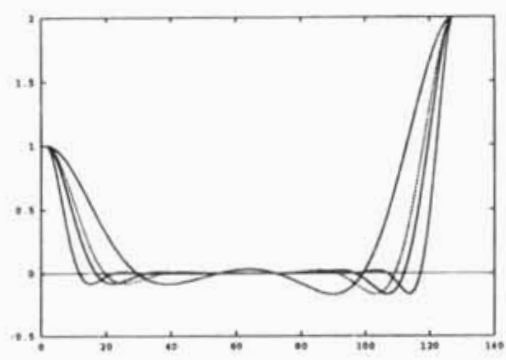

(a)

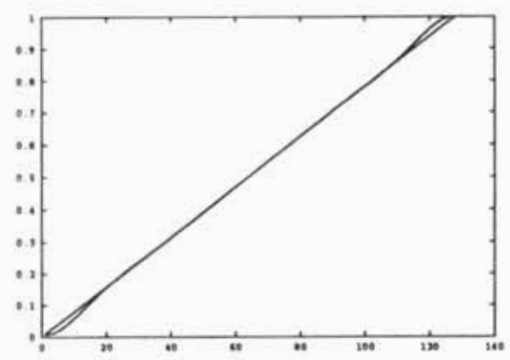

(b)

Figure 2: Solutions of equ (2) : (a) Some solutions with $\tilde{f}(x)=\delta(x)+2 \delta(x-1)$; (b) Stationnary solution with $\tilde{f}(x)=x$, (in the 2 cases the 2 extremal points of the $x$ domain are fixed and the derivatives on these points are 0 )

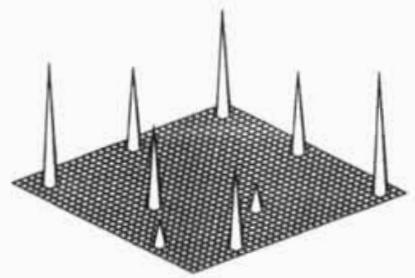

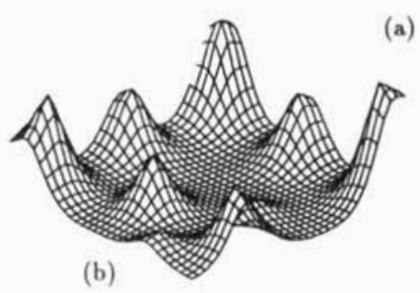

(b)

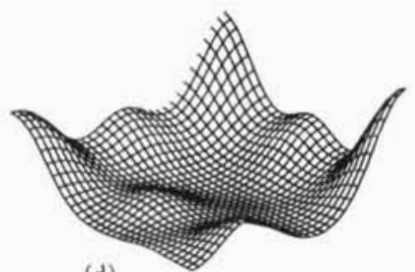

(d)

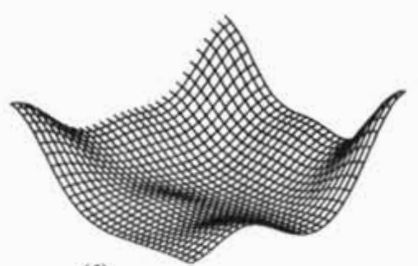

(f)

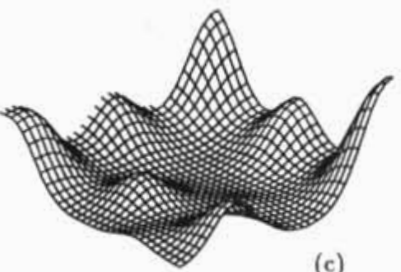

(c)

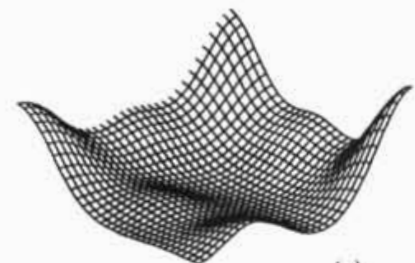

(e)

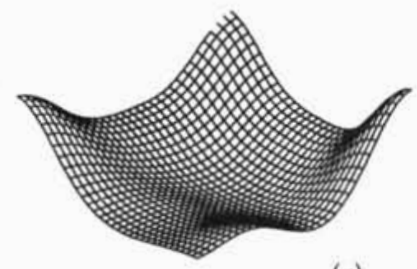

(g)

Figure 3: (a) Original points ; (b) (c) (d)(e)(f)(g) Intermediary interpolation steps . 


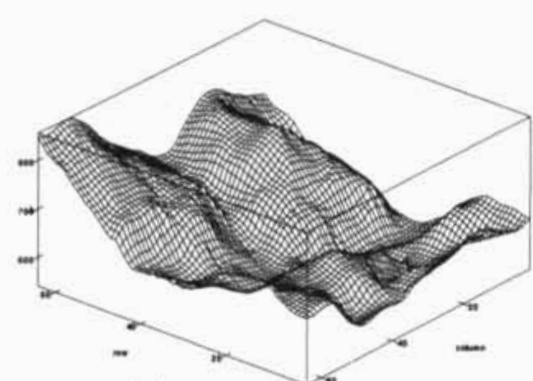

(a)

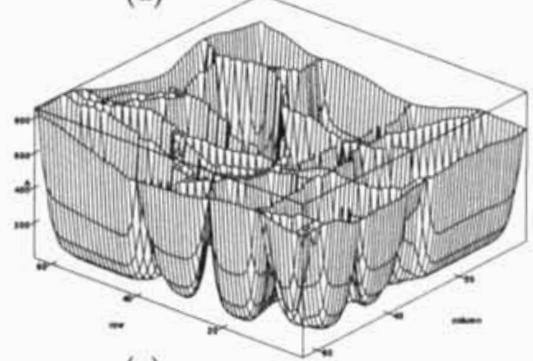

(c)

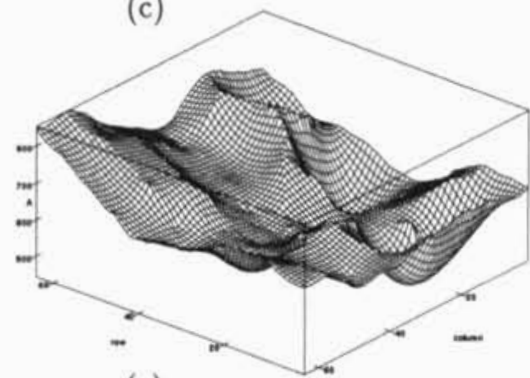

(e)

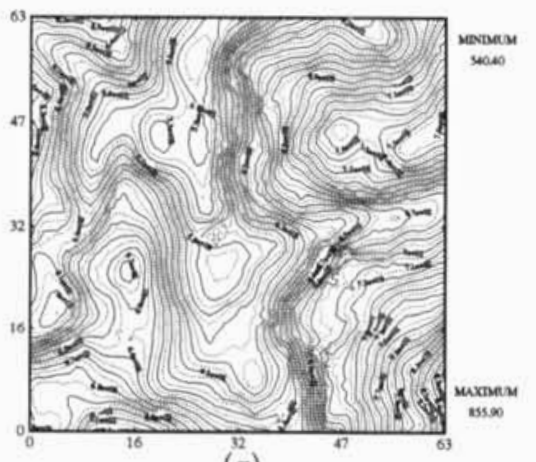

(g)

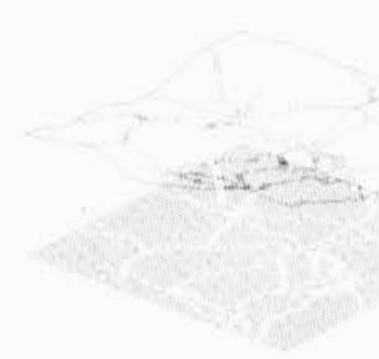

(b)

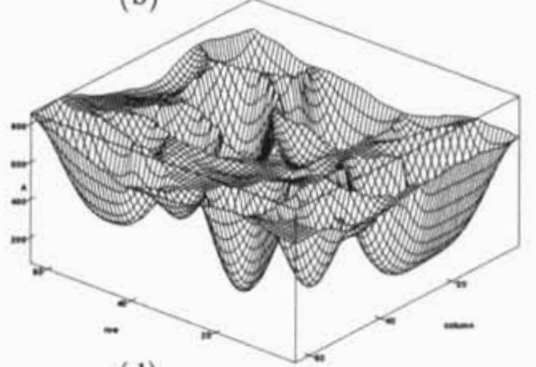

(d)

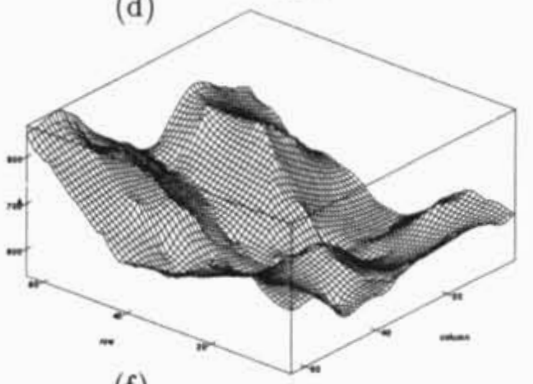

(f)

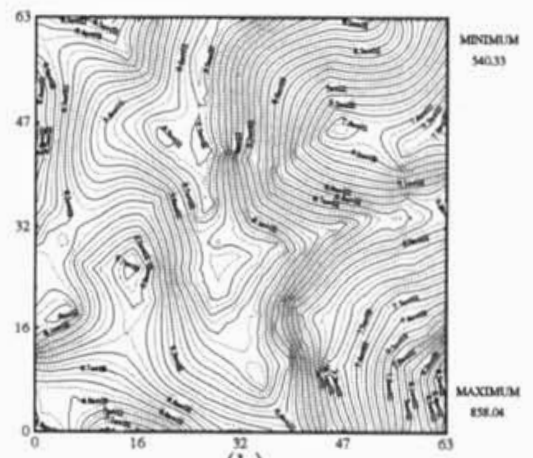

(h)

Figure 4: (a) Original DEM; (b) Ridge line, stream network and boundary points ( 814 points $\sim 20 \%$ of the original DEM points); (c) 2 iterations; (d) 20 iterations; (e) 80 iterations; (f) 150 iterations. (we have taken $\alpha=2$ ). (g) Contour lines of the DEM given in (a); (h) Contour lines of the reconstructed DEM. 